\title{
Correlates of dietary energy misreporting among European adolescents: the Healthy Lifestyle in Europe by Nutrition in Adolescence (HELENA) study
}

\author{
Silvia Bel-Serrat ${ }^{1,2 *}$, Cristina Julián-Almárcegui ${ }^{1}$, Marcela González-Gross ${ }^{3}$, Theodora Mouratidou ${ }^{1}$, \\ Claudia Börnhorst ${ }^{4}$, Evangelia Grammatikaki ${ }^{5,6}$, Mathilde Kersting ${ }^{7}$, Magdalena Cuenca-García ${ }^{8,9}$, \\ Frederic Gottrand $^{10}$, Dénes Molnár ${ }^{11}$, Lena Hallström ${ }^{12}$, Jean Dallongeville ${ }^{13}$, Maria Plada ${ }^{14}$,
} Romana Roccaldo $^{15}$, Kurt Widhalm ${ }^{16}$, Luis A. Moreno ${ }^{1}$, Yannis Manios ${ }^{6}$, Stefaan De Henauw ${ }^{4}$, Catherine Leclercq ${ }^{15}$, Stefanie Vandevijvere ${ }^{17,18}$, Sandrine Lioret ${ }^{19}$, Bernard Gutin ${ }^{20}$ and Inge Huybrechts ${ }^{2,5}$

${ }^{1}$ Growth, Exercise, Nutrition and Development (GENUD) Research Group, University of Zaragoza, Pedro Cerbuna 12, 50009 Zaragoza, Spain

${ }^{2}$ Dietary Exposure Assessment (DEX) Group, International Agency for Research on Cancer, 150 Cours Albert Thomas, 69372 Lyon, France

${ }^{3}$ ImFINE Research Group, Department of Health and Human Performance, Technical University of Madrid, Avenida Martin Fierro 7, 28040 Madrid, Spain

${ }^{4}$ Leibniz Institute for Prevention Research and Epidemiology-BIPS, Achterstr. 30, 28359 Bremen, Germany

${ }^{5}$ Department of Public Health, Ghent University, P/A UZ 4K3, De Pintelaan 185, B-9000 Ghent, Belgium

${ }^{6}$ Department of Nutrition and Dietetics, Harokopio University, 70 El. Venizelou, Kallithea 17671, Athens, Greece

${ }^{7}$ Research Institute of Child Nutrition Dortmund, Heinstueck 11, D-44225 Dortmund, Germany

${ }^{8}$ Department of Physiology, School of Medicine, University of Granada, Avenida Madrid 11, 18012 Granada, Spain

${ }^{9}$ Department of Physical Education, Faculty of Education Science, University of Cadiz, Avenida República Saharaui s/n, 11519 Cádiz, Spain

${ }^{10}$ LIRIC UMR995, Faculté de Médecine, University of Lille 2, CHRU Lille, Bd Pr Leclercq, Hôpital Cardiologique, 59037 Lille, France

${ }^{11}$ Department of Pediatrics, University of Pécs, József A 7, 7623 Pécs, Hungary

${ }^{12}$ Unit for Preventive Nutrition, Department of Biosciences and Nutrition, Karolinska Institutet, SE 14157 Huddinge, Sweden

${ }^{13}$ Institut Pasteur de Lille, 1 rue du Prof. Calmette, 59019 Lille, France

${ }^{14}$ Preventive Medicine \& Nutrition Unit, University of Crete School of Medicine, Heraklion, Crete, GR-71003, Greece

${ }^{15}$ Council for Agricultural Research and Economics (CREA), Research Center for Food and Nutrition, Via Ardeatina 546, 00178 Rome, Italy

${ }^{16}$ Division of Clinical Nutrition and Prevention, Department of Pediatrics, Medical University of Vienna, Währinger Gürtel 18-20, A-1090 Vienna, Austria

${ }^{17}$ Department of Public Health and Surveillance, Scientific Institute of Public Health, Rue Juliette Wytsmanstraat 14, 1050 Brussels, Belgium

${ }^{18}$ School of Population Health, University of Auckland, Tamaki Campus, Building 730, Cnr Morrin Road E Merton Road, Glen Innes, Private Bag 92019, Auckland, New Zealand

${ }^{19}$ INSERM, UMR1153 Epidemiology and Biostatistics Sorbonne Paris Cité Center (CRESS), Early ORigin of the Child's Health And Development Team (ORCHAD), 16, avenue Paul Vaillant Couturier, 94807 Villejuif Cedex, France

${ }^{20}$ Department of Pediatrics, Medical College of Georgia, 1120 15th Street, BT-1852 Augusta, GA 30912, USA

(Submitted 28 August 2015 - Final revision received 13 January 2016 - Accepted 15 January 2016 - First published online 18 February 2016)

\section{Abstract}

This study examined the correlates of dietary energy under-reporting (UR) and over-reporting (OV) in European adolescents. Two selfadministered computerised 24-h dietary recalls and physical activity data using accelerometry were collected from 1512 adolescents aged 12.5-17.5 years from eight European countries. Objective measurements of height and weight were obtained. BMI was categorised according to Cole/International Obesity Task Force (IOTF) cut-off points. Diet-related attitudes were assessed via self-administered questionnaires.

Abbreviations: 24-HDR, 24-h dietary recall; EI, energy intake; HELENA-CSS, Healthy Lifestyle in Europe by Nutrition in Adolescence Cross-Sectional Study; MVPA, moderate-to-vigorous physical activity; OV, over-reporting; PA, physical activity; SES, socio-economic status; TEE, total energy expenditure; UR, under-reporting.

*Corresponding author: S. Bel-Serrat, email sbel@unizar.es 
Reported energy intake (EI) was compared with predicted total energy expenditure to identify UR and OV using individual physical activity objective measures. Associations between misreporting and covariates were examined by multilevel logistic regression analyses. Among all, $33.3 \%$ of the adolescents were UR and $15.6 \%$ were OV when considering mean EI. Overweight (OR 3.25; 95\% CI 2.01, 5.27) and obese (OR $4.31 ; 95 \%$ CI 1.92, 9.65) adolescents had higher odds for UR, whereas underweight individuals were more likely to over-report (OR 1.67; $95 \%$ CI 1.01, 2.76). Being content with their own figures (OR 0.61; $95 \%$ CI $0.41,0 \cdot 89$ ) decreased the odds for UR, whereas frequently skipping breakfast (OR 2.14; $95 \%$ CI 1.53, 2.99) was linked with higher odds for UR. Those being worried about gaining weight (OR 0.55; $95 \%$ CI 0.33 , 0.92) were less likely to OV. Weight status and psychosocial weight-related factors were found to be the major correlates of misreporting. Misreporting may reflect socially desirable answers and low ability to report own dietary intakes, but also may reflect real under-eating in an attempt to lose weight or real over-eating to reflect higher intakes due to growth spurts. Factors influencing misreporting should be identified in youths to clarify or better understand diet-disease associations.

Key words: Misreporting: Under-reporting: Obesity: Adolescence: Healthy Lifestyle in Europe by Nutrition in Adolescence study

Accurate measurement of food and energy intake (EI) is the basis of studies that focus on associations between diet and health $^{(1)}$. Dietary assessment on its own is associated with several concerns regarding validity, mostly because it relies on self-report and/or proxy-reported measurements ${ }^{(2)}$. No gold standard for the evaluation of reported dietary intake exists ${ }^{(3)}$; many diet studies are faced with the reporting of implausible EI in both directions, under-reporting (UR) and over-reporting $(\mathrm{OV})^{(1)}$, a widely acknowledged limitation of dietary assessment methods.

EI misreporting is characterised by reports of habitual EI, which are implausibly low or high, that is, UR or OV, respectively, when compared with the energy requirements estimated using objective methods of energy expenditure, such as the doubly labelled water (DLW) technique or prediction equations ${ }^{(4)}$. This implies the presence of systematic bias and differential misreporting in dietary intake assessments, which may attenuate, or even reverse, the directions of the associations under study ${ }^{(5)}$. This problem may also lead to an inadequate estimation of the prevalence of nutrient deficiencies. For that reason, intentional dietary misreporting represents a major concern in studies that monitor dietary intake at the population level and/or evaluate diet-health associations $^{(6,7)}$.

Identification of misreporting in any of its forms, that is, UR and/or OV, and its characteristics is thus crucial to the appropriate interpretation of nutritional data ${ }^{(3)}$. Different studies have investigated the factors associated with intentional misreporting; however, a consensus is still lacking for various reasons ${ }^{(5)}$. BMI in particular has been repeatedly linked to misreporting in adults $^{(1)}$, but also among children and adolescents ${ }^{(5)}$. Other factors found to be associated with EI misreporting include age, sex, socio-economic status (SES), health consciousness, proxyreporting, cultural variations and psychological differences ${ }^{(1)}$.

As with adults, inaccurate energy reporting also occurs among young populations ${ }^{(5,8)}$. Adolescents, unlike children, have full cognitive capability to provide self-reported dietary data; however, adolescence is characterised by increasingly greater food requirements, unstructured eating patterns, rapidly changing food habits and more frequent out-of-home eating ${ }^{(9)}$. These factors, along with a possibly reduced level of interest to recall their own intake, might lead to less motivation, forgetfulness and lack of compliance, and thus to a reduced reporting accuracy ${ }^{(9)}$

As has been the case for similar surveys, the Healthy Lifestyle in Europe by Nutrition in Adolescence Cross-Sectional Study
(HELENA-CSS) is also susceptible to EI misreporting ${ }^{(10)}$. Therefore, the present study assessed UR and OV among European freeliving adolescents participating in this study. Available information on misreporting and its correlates in European adolescents is still scarce. Therefore, several factors potentially associated with dietary misreporting according to previous literature - that is, socio-demographic indicators, lifestyle variables, weight status and weight- and diet-related attitudes - were investigated in this sample of European adolescents to shed light on this underinvestigated topic in youth populations.

\section{Methods}

\section{Subjects and study design}

The HELENA-CSS obtained standardised, reliable and comparable data from a random sample of European adolescents on a broad battery of relevant nutrition and health-related parameters $^{(10,11)}$. Data collection took place during 2006 and 2007 in ten European cities: Athens (inland city) and Heraklion (Mediterranean island city) in Greece, Dortmund in Germany, Ghent in Belgium, Lille in France, Pécs in Hungary, Rome in Italy, Stockholm in Sweden, Vienna in Austria and Zaragoza in Spain. A detailed description of the sampling and recruitment approaches, standardisation and harmonisation processes, data collection, analysis strategies and quality control activities has been published elsewhere ${ }^{(10-12)}$. Written informed consent was obtained from all adolescents and their parents or guardians. The study was conducted according to the guidelines laid down in the Declaration of Helsinki, and all the procedures involving human subjects/patients were approved by the Human Ethics Committees of the centres involved ${ }^{(12)}$.

A total of 3528 adolescents (52.3\% females) aged $12 \cdot 5-17 \cdot 5$ years were recruited from randomly selected schools in each city. The mean participation rate within the HELENA-CSS was $67 \%$, which can be considered acceptable, given the demanding nature of this epidemiological study. The response for all the questionnaires included in the database was more than $80 \%$. For the purposes of this study, only those adolescents who completed two 24-h dietary recalls (24-HDR) and had objectively measured accelerometer data were included ( $n$ 1512). Owing to logistical reasons, energy and nutrient intake information from the participants from Greece and Hungary was available for only $1 \mathrm{~d}$. Therefore, only eight HELENA-CSS centres were included in this study. Excluded adolescents 
( $n$ 2016) weighed significantly more $(60 \cdot 1 v .57 \cdot 8 \mathrm{~kg} ; P<0 \cdot 05)$ and had significantly higher BMI mean $\left(21.7 v .21 \cdot 0 \mathrm{~kg} / \mathrm{m}^{2}\right)$ compared with those included in this study ( $n$ 1512).

\section{Socio-economic status, educational level and parental weight perception}

A self-reported questionnaire was used to collect data on living conditions, family structure and employment status, occupation and educational level of both parents ${ }^{(13)}$. The Family Affluence Scale was used as an indicator of affluence, based on the concept of material conditions of the household in which adolescents lived $^{(14)}$. Family affluence was determined from a set of items including car ownership, bedroom occupancy, home computers and Internet access. The adolescents reported the educational level of their parents (dummy: low/medium $v$. high). Their perception of parental weight (dummy: overweight/obese $v$. normal weight/thin/very thin) was also recorded.

\section{Sedentary and sleep behaviours}

The average minutes per day in which the adolescents were engaged in two sedentary behaviours (TV viewing and playing with video games) were estimated by means of a selfadministered questionnaire previously found to demonstrate good reliability ${ }^{(15)}$. Habitual sleep time $(\mathrm{h} / \mathrm{d})$, as estimated by a self-reported questionnaire, was defined as the average of sleep duration during weekdays and weekend days.

\section{Physical activity}

Physical activity (PA) assessment in the HELENA-CSS study is described elsewhere ${ }^{(16)}$. Uni-axial accelerometers (Actigraph MTI, model GT1M; Manufacturing Technology Inc.) were used to objectively measure PA. Adolescents were asked to wear the accelerometer for 7 consecutive days during all waking hours, except for water-based activities. At least $3 \mathrm{~d}$ of recording, with a minimum of $8 \mathrm{~h}$ of registration/d, was set as an inclusion criterion. The time-sampling interval was set at $15 \mathrm{~s}$, and bouts of $\geq 20 \mathrm{~min}$ of consecutive zero counts were deleted from the data sets. Total PA was expressed as total counts recorded, divided by total daily registered time (counts per min). The time spent at moderate-to-vigorous PA (MVPA) ( $>3$ metabolic equivalents) was calculated on the basis of the following cut-off points $-\geq 2000$ counts per min for moderate PA and $\geq 4000$ counts per min for vigorous $\mathrm{PA}^{(16)}$ - and was presented as the average time per day $(\mathrm{min} / \mathrm{d})$. Total energy expenditure (TEE, $\mathrm{kJ} / \mathrm{d}$ or $\mathrm{kcal} / \mathrm{d}$ ) was estimated from activity counts using the equation of Ekelund et $a l .{ }^{(17)}$ already validated in youth:

$$
\begin{aligned}
(- & \text { Sex } \times 380 \cdot 9)+(1.177 \times \text { counts per min }) \\
& +(21.1 \times \text { weight }(\mathrm{kg}))+706,
\end{aligned}
$$

where sex was coded as 0 in boys and 1 in girls. This formula from the study of Ekelund et al. ${ }^{(17)}$ was derived from multiple stepwise regression analyses where the best regression equation explained $60 \%$ of the variation in TEE and included sex, activity counts and body weight. The cross-validation study showed no significant differences between predicted and measured TEE by the developed prediction equation; thus, it was judged to provide valid data for assessing TEE in youth.

\section{Physical examination}

Anthropometric measurements were obtained following a standardised protocol ${ }^{(18)}$. Weight and height were measured in underwear and barefoot using an electronic scale (type SECA 861; Seca Ltd) and a stadiometer (type SECA 225; Seca Ltd). BMI was calculated as body weight in kilograms divided by the square of height in metres and was additionally categorised according to Cole et al. $^{(19,20)}$.

\section{Diet-related attitudes}

The validated 'eating attitudes and weight problems inventory' designed for adolescents ${ }^{(21)}$ was used to allow cross-cultural comparisons in dietary attitudes and behaviours, as well as to link the data to food habits ${ }^{(22)}$. The following statements were entered in the analysis: 'I'm very worried about gaining weight', 'I dread being fat', 'I am constantly aware that I weigh too much', 'I often eat less than I would like to not gain weight', 'I deliberately have small portions to not gain weight', 'I try to eat as little as possible so that I do not gain weight', 'I'm content with my figure' and 'My parents think I'm too fat'. Responses were re-coded into two categories (dummy: does not apply/ seldom $v$. occasionally/always applies). Breakfast consumption was assessed by the 'food choices and preferences' questionnaire ${ }^{(23)}$. The statement 'I often skip breakfast' was also assessed (dummy: strongly/moderately/slightly disagree $v$. strongly/moderately/slightly agree).

\section{Dietary intake}

Following recommendations of the 'European Food Consumption Survey Method' project, two non-consecutive 24-HDR, within a time span of 2 weeks, were completed by the adolescents ${ }^{(24)}$. Assessment was performed by a computer-based tool for self-reported 24-HDR, HELENA-Dietary Assessment Tool (DIAT), based on a previous version developed for Flemish adolescents, shown to provide valid measurements of dietary intake compared with an interview by a dietitian ${ }^{(25)}$. Dietary intake referred to the day before the administration and was divided into six meal occasions. For each occasion, the user was invited to select all the consumed food items and beverages from a standardised food list. Information on quantities was collected using household measurements or pictures of portion sizes. The self-administration took place during school time in a computer classroom where the pupils completed the programme autonomously while fieldworkers were present to give assistance if necessary ${ }^{(25)}$. No information on Fridays and Saturdays was available.

The German Food Code and Nutrition Database (Bundeslebensmittelschlüssel (BLS), version II.3.1, 2005) was used to calculate energy and nutrient intakes; the BLS is the most complete food composition database across Europe in terms of nutrients and food items ${ }^{(26,27)}$. EI was estimated in $\mathrm{kJ} / \mathrm{d}$ 
or $\mathrm{kcal} / \mathrm{d}$ and macronutrient intakes (fat, protein and carbohydrate) were expressed in $\mathrm{g} / \mathrm{d}$. Subsequently, intakes of each macronutrient were converted into percentage of total EI.

\section{Energy intake misreporting: under-reporting and over-reporting}

UR and OV were calculated according to the approach proposed by Huang et $a l .{ }^{(28)}$. The method relies on the direct comparison of reported EI and predicted TEE, based on the principle that EI is equal to TEE, assuming weight stability. The approach uses \pm 1 SD cut-off points to statistically compare reported EI with predicted TEE. A report is excluded if \%EI/TEE is outside the $\pm 1 \mathrm{SD}$ range. A \%EI/TEE outside this range indicates that EI is too low $(<-1 \mathrm{sD})$ or too high $(>+1 \mathrm{SD})$ to represent the habitual intake and that the reported EI is therefore implausible. The \pm 1 SD cut-off points were calculated by means of the following equation:

$$
\pm 1 \mathrm{SD}=\sqrt{ }\left(\left(\mathrm{CV}_{\mathrm{rEI}}^{2} / d\right)+\mathrm{CV}_{\mathrm{pTEE}}^{2}+\mathrm{CV}_{\mathrm{mTEE}}^{2}\right) .
$$

The equation accounts for intra-individual variation in EI reporting $\left(\mathrm{CV}_{\mathrm{rEI}}\right)$ over the number of days $(d)$ of intake, the error in the equations for predicted TEE $\left(\mathrm{CV}_{\text {PTEE}}\right.$, which includes the errors of the parameters in those equations, including physical activity level) and measurement error and day-to-day biological variation in TEE $\left(\mathrm{CV}_{\mathrm{mTEE}}\right)^{(28)}$. Number of days were $d=2$ for the mean of two interviews and $d=1$ when the cut-off points were calculated for one interview day. The $\mathrm{CV}_{\mathrm{rEI}}$ and the $\mathrm{CV}_{\mathrm{pTEE}}$ were calculated separately by sex based on the HELENA-CSS data ${ }^{(29)}$. The $\mathrm{CV}_{\text {mTEE }}$ was set to $8 \cdot 2 \%$, as estimated from DLW measurements ${ }^{(30)}$. Adolescents were classified as under-reporters, plausible reporters or over-reporters according to these cut-off values. All values and sex-specific cut-off points to estimate UR and OV are presented in Table 1.

\section{Statistical analysis}

The statistical software package Stata version 12.0 (StataCorp LP), was used to perform the analyses, and the threshold for statistical significance was set at $P \leq 0 \cdot 05$. Characteristics of the study sample are presented as medians and percentiles for continuous variables and as percentages for categorical variables. Multilevel logistic regression analysis, with study centre as the random intercept, was performed to investigate factors associated with misreporting, considering UR and OV as the outcome variables (reference category: plausible reporters). Analysis was conducted at a first step for each potential correlate of UR and OV, adjusting for age and sex. At a second step, those variables with $P<0.20$ were entered simultaneously in the same model with UR and OV as the outcome variables.

\section{Results}

\section{Energy intake misreporting}

Table 2 shows the degree of misreporting on day 1 and day 2, separately. The degree of UR was higher for the second 24-HDR (29.5\%) than for the first one (26.5\%), whereas adolescents over-reported more on the first 24-HDR (14.6 v. 14.0\%). When assessing misreporting for mean EI of both interview days, 33.3 and $15.6 \%$ of the adolescents were categorised as underreporters and over-reporters, respectively (Table 3 ).

The degree of UR and OV separately by sex and weight status is displayed in Table 3. The percentage of UR was higher for girls $(35.7 \%)$ than for boys $(30 \cdot 4 \%)$. However, boys overreported slightly more $(15.8 \%)$ than girls $(15.5 \%)$. The degree of UR was higher among overweight (59.3\%) and obese (66.2\%) adolescents compared with normal-weight (27.6\%) and underweight adolescents (13.0\%) and increased with increasing BMI categories. On the contrary, underweight adolescents over-reported more (33.3\%), followed to a lesser extent by normal-weight (16.9\%), overweight (5.5\%) and obese $(2 \cdot 8 \%)$ adolescents.

\section{Characteristics of the study sample}

Characteristics of the study sample are presented in Table 4 for the total study sample and stratified by reporting group (UR, plausible reporting and OV). In the UR and OV groups, a higher percentage of adolescents were females. Adolescents classified as under-reporters stated more that they were worried about gaining weight, dreaded being fat and skipped breakfast. Compared with plausible reporters and over-reporters, adolescents in the UR group had higher median BMI, MVPA, TEE and contribution of protein and carbohydrate intakes to EI. Overreporters showed higher median of EI, screen time engagement and contribution of fat to EI.

\section{Correlates of under-reporting}

The results of the first step of the multilevel logistic regression analysis are shown in Table 5, where the model was only

Table 1. Reference and cut-off values used to identify misreporting among adolescents

\begin{tabular}{|c|c|c|c|c|c|c|}
\hline \multirow[b]{2}{*}{ Sex } & \multirow[b]{2}{*}{$n$} & \multirow[b]{2}{*}{$\mathrm{CV}_{\mathrm{rEI}} \dagger(\%)$} & \multirow[b]{2}{*}{$\mathrm{CV}_{\mathrm{pER}} \neq(\%)$} & \multirow[b]{2}{*}{$\mathrm{CV}_{\text {mTEE }} \S(\%)$} & \multicolumn{2}{|c|}{ Cut-off values $(\%)^{\star}$} \\
\hline & & & & & One interview & Mean two interviews \\
\hline Boys & 684 & 32.1 & 13.8 & 8.2 & 36 & 28 \\
\hline Girls & 828 & $32 \cdot 0$ & $14 \cdot 2$ & 8.2 & 36 & 28 \\
\hline
\end{tabular}

$\mathrm{El}$, energy intake; ER, energy requirement; TEE, total energy expenditure.

* Cut-off values calculated according to Huang et al. ${ }^{(28)}$

$\dagger$ Intra-individual variation of El.

$\ddagger$ Error in predicted energy expenditure requirements.

$\S$ Day-to-day variation and measurement error for TEE based on the doubly labelled water technique ${ }^{(30)}$. 
Table 2. Energy reporting for 24-h dietary recalls on interview days 1 and 2

(Numbers and percentages)

\begin{tabular}{|c|c|c|c|c|c|c|c|c|c|c|c|c|}
\hline \multirow[b]{4}{*}{ Sex } & \multicolumn{12}{|c|}{ One interview } \\
\hline & \multicolumn{6}{|c|}{ Day 1} & \multicolumn{6}{|c|}{ Day 2} \\
\hline & \multicolumn{2}{|c|}{ Under-reporters } & \multicolumn{2}{|c|}{ Plausible reporters } & \multicolumn{2}{|c|}{ Over-reporters } & \multicolumn{2}{|c|}{ Under-reporters } & \multicolumn{2}{|c|}{ Plausible reporters } & \multicolumn{2}{|c|}{ Over-reporters } \\
\hline & $n$ & $\%$ & $n$ & $\%$ & $n$ & $\%$ & $n$ & $\%$ & $n$ & $\%$ & $n$ & $\%$ \\
\hline Total & 400 & $26 \cdot 5$ & 891 & 58.9 & 221 & $14 \cdot 6$ & 446 & 29.5 & 854 & 56.5 & 212 & $14 \cdot 0$ \\
\hline Boys & 161 & 23.5 & 414 & 60.5 & 109 & $15 \cdot 9$ & 193 & $28 \cdot 2$ & 398 & 58.2 & 93 & $13 \cdot 6$ \\
\hline Girls & 239 & 28.9 & 477 & 57.6 & 112 & 13.5 & 253 & 30.6 & 456 & 55.1 & 119 & 14.4 \\
\hline
\end{tabular}

Table 3. Energy reporting by sex and weight status (Numbers and percentages)

\begin{tabular}{|c|c|c|c|c|c|c|}
\hline & \multicolumn{2}{|c|}{ Under-reporters } & \multicolumn{2}{|c|}{ Plausible reporters } & \multicolumn{2}{|c|}{ Over-reporters } \\
\hline & $n$ & $\%$ & $n$ & $\%$ & $n$ & $\%$ \\
\hline All sample & 504 & $33 \cdot 3$ & 772 & $51 \cdot 1$ & 236 & $15 \cdot 6$ \\
\hline \multicolumn{7}{|l|}{ Sex } \\
\hline Boys & 208 & $30 \cdot 4$ & 368 & 53.8 & 108 & $15 \cdot 8$ \\
\hline Girls & 296 & 35.7 & 404 & $48 \cdot 8$ & 128 & $15 \cdot 5$ \\
\hline \multicolumn{7}{|l|}{ Weight status* } \\
\hline Underweight & 14 & 13.0 & 58 & 53.7 & 36 & 33.3 \\
\hline Normal weight & 303 & $27 \cdot 6$ & 609 & 55.5 & 185 & $16 \cdot 9$ \\
\hline Overweight & 140 & $59 \cdot 3$ & 83 & $35 \cdot 2$ & 13 & 5.5 \\
\hline Obese & 47 & $66 \cdot 2$ & 22 & 31.0 & 2 & $2 \cdot 8$ \\
\hline
\end{tabular}

${ }^{*} \mathrm{BMI}$ categories as described by Cole et al. ${ }^{(19,20)}$.

adjusted for sex and age. No significant associations were found between UR and OV and either age or sex. Under-reporters were less likely to be underweight and reported a higher contribution of fat intake to EI. On the other hand, under-reporters were more likely to be overweight or obese, perceived their own mothers as overweight/obese, had higher MVPA levels, reported higher contributions of protein and carbohydrate intakes to EI, were worried about gaining weight, dreaded being fat, were constantly aware that they weighed too much, reported eating less often than they liked, reported eating deliberately small portions and trying to eat as little as possible to not gain weight. Underreporters skipped breakfast more often, and were more likely to report that their parents perceived them as being too fat. Lower risk of UR was observed among those adolescents who were content with their figure. Over-reporters were more likely to be underweight and had higher screen time, whereas they were less likely to be overweight or obese and reported lower contribution of protein intake to EI. Those who were worried about gaining weight, dreaded being fat, were constantly aware that they weighed too much and tried to eat as little as possible to not gain weight were less likely to over-report.

Most of the associations found in the previous model did not remain significant when the model was simultaneously adjusted for other covariates potentially related to UR or OV (Table 6). Overweight (OR 3.25; $95 \%$ CI 2.01, 5.27) and obese (OR 4.31; $95 \%$ CI $1.92,9.65)$ adolescents were more likely to under-report and underweight adolescents were more likely to over-report (OR 1.67; $95 \%$ CI 1.01, 2.76). Increasing screen time reduced the odds for UR (OR 0.997; 95\% CI 0.995, 0.999) and increased the odds for OV (OR 1.003; 95\% CI 1.001, 1.005). The associations observed previously between MVPA and UR (OR 1.02; $95 \%$ CI 1.01, 1.02) remained significant. Besides, OV and MVPA levels were significantly associated (OR 0.991; 95\% CI $0.983,0.999)$. The same applied for the contribution of protein intake to EI for both UR (OR 1.08; $95 \%$ CI 1.02, 1.14) and OV (OR 0.87; 95\% CI 0.82, 0.93) and for the contribution of carbohydrate intake to EI (OR 1.06; 95\% CI 1.03, 1.09) among under-reporters. Considering diet-related attitudes, hardly any of the associations previously observed persisted in the second model. Those who were content with their figure were less likely to under-report (OR 0.61; 95\% CI 0.41, 0.89), and those who skipped breakfast were more likely to under-report (OR 2.14; $95 \%$ CI $1.53,2.99)$. On the other hand, those who were very worried about gaining weight were less likely to overreport (OR 0.55; $95 \%$ CI 0.33, 0.92).

\section{Discussion}

To the best of our knowledge, this is the first study assessing misreporting and its correlates in a large sample of adolescents from several cities across Europe, offering the opportunity to examine these factors among culturally diverse populations. A review on implausible EI in children and adolescents revealed that the prevalence of misreporting among studies ranged from 2 to $85 \%$ for UR and from 3 to $46 \%$ for $\mathrm{OV}^{(31)}$. As already acknowledged by Börnhost et al. ${ }^{(8)}$, the large variability observed in the occurrence of UR can be explained by different methodologies (dietary assessment tools and number of assessment days), cut-off values applied, respondent 
Table 4. Descriptive characteristics of the sample by reporting group (Medians and 25th-75th percentiles)

\begin{tabular}{|c|c|c|c|c|c|c|c|c|}
\hline & \multicolumn{2}{|c|}{ Under-reporters } & \multicolumn{2}{|c|}{ Plausible reporters } & \multicolumn{2}{|c|}{ Over-reporters } & \multicolumn{2}{|c|}{ Total study group } \\
\hline & Median & $\begin{array}{l}\text { 25th-75th } \\
\text { percentile }\end{array}$ & Median & $\begin{array}{l}\text { 25th-75th } \\
\text { percentile }\end{array}$ & Median & $\begin{array}{l}\text { 25th-75th } \\
\text { percentile }\end{array}$ & Median & $\begin{array}{l}\text { 25th-75th } \\
\text { percentile }\end{array}$ \\
\hline $\begin{array}{l}\text { Age (years) } \\
\text { Weight }(\mathrm{kg}) \\
\text { Height }(\mathrm{cm}) \\
\text { BMI }\left(\mathrm{kg} / \mathrm{cm}^{2}\right) \\
\text { Screen time }(\mathrm{min} / \mathrm{d}) \\
\text { Sleep time }(\mathrm{h} / \mathrm{d}) \\
\text { MVPA }(\mathrm{min} / \mathrm{d}) \\
\text { EI (kJ/d) } \\
\text { EI (kcal/d) } \\
\mathrm{TEE}^{*}(\mathrm{~kJ} / \mathrm{d}) \\
\mathrm{TEE}(\mathrm{kcal} / \mathrm{d}) \\
\text { Proteins }(\% \text { of total El) } \\
\text { Fat }(\% \text { of total El) } \\
\text { Carbohydrates }(\% \text { of total El) }\end{array}$ & $\begin{array}{r}14 \cdot 5 \\
60 \cdot 1 \\
164 \cdot 6 \\
22 \cdot 2 \\
107 \cdot 1 \\
8 \cdot 0 \\
57 \cdot 7 \\
5843 \cdot 6 \\
1398 \cdot 0 \\
10617 \cdot 6 \\
2540 \cdot 1 \\
16 \cdot 4 \\
32 \cdot 9 \\
50 \cdot 6\end{array}$ & $\begin{array}{c}13 \cdot 6-15 \cdot 6 \\
52 \cdot 8-69 \cdot 3 \\
159 \cdot 1-170 \cdot 2 \\
19 \cdot 9-24 \cdot 7 \\
57 \cdot 9-167 \cdot 1 \\
7 \cdot 0-9 \cdot 0 \\
43 \cdot 5-73 \cdot 9 \\
4737 \cdot 0-7074 \cdot 6 \\
1147 \cdot 6-1692 \cdot 5 \\
9313 \cdot 5-12175 \cdot 5 \\
2228 \cdot 1-2912 \cdot 8 \\
14 \cdot 2-19 \cdot 0 \\
28 \cdot 8-37 \cdot 0 \\
45 \cdot 3-55 \cdot 1\end{array}$ & $\begin{array}{r}14 \cdot 7 \\
55 \cdot 4 \\
165 \cdot 3 \\
20 \cdot 0 \\
107 \cdot 1 \\
8 \cdot 0 \\
53 \cdot 0 \\
9431 \cdot 3 \\
2256 \cdot 3 \\
10091 \cdot 4 \\
2414 \cdot 2 \\
16 \cdot 0 \\
33 \cdot 9 \\
48 \cdot 6\end{array}$ & $\begin{array}{c}13 \cdot 7-15 \cdot 6 \\
48 \cdot 8-62 \cdot 5 \\
159 \cdot 3-172 \cdot 0 \\
18 \cdot 4-21 \cdot 9 \\
68 \cdot 6-182 \cdot 1 \\
7 \cdot 5-9 \cdot 0 \\
40 \cdot 9-69 \cdot 6 \\
8144 \cdot 3-11242 \cdot 9 \\
1948 \cdot 4-2689 \cdot 7 \\
8676 \cdot 4-11242 \cdot 9 \\
2075 \cdot 7-2746 \cdot 7 \\
14 \cdot 0-18 \cdot 2 \\
37 \cdot 9-30 \cdot 0 \\
43 \cdot 6-53 \cdot 0\end{array}$ & $\begin{array}{r}14 \cdot 7 \\
52 \cdot 1 \\
165 \cdot 5 \\
18 \cdot 9 \\
124 \cdot 3 \\
8.0 \\
51 \cdot 2 \\
14858 \cdot 2 \\
3554 \cdot 6 \\
9390 \cdot 8 \\
2246 \cdot 3 \\
14 \cdot 9 \\
34 \cdot 3 \\
48 \cdot 1\end{array}$ & $\begin{array}{c}13 \cdot 6-15 \cdot 5 \\
45 \cdot 8-58 \cdot 5 \\
158 \cdot 3-172 \cdot 1 \\
17 \cdot 7-20 \cdot 5 \\
75 \cdot 0-207 \cdot 9 \\
7 \cdot 5-9 \cdot 0 \\
37 \cdot 0-68 \cdot 5 \\
12373 \cdot 2-18171 \cdot 3 \\
2960 \cdot 1-4347 \cdot 2 \\
8105 \cdot 4-10940 \cdot 7 \\
1939 \cdot 1-2617 \cdot 4 \\
12 \cdot 8-17 \cdot 4 \\
30 \cdot 6-37 \cdot 9 \\
44 \cdot 0-52 \cdot 7\end{array}$ & $\begin{array}{r}14 \cdot 6 \\
56 \cdot 3 \\
165 \cdot 1 \\
20 \cdot 4 \\
107 \cdot 1 \\
8 \cdot 0 \\
53 \cdot 9 \\
8704 \cdot 4 \\
2082 \cdot 4 \\
10172 \cdot 5 \\
2433 \cdot 6 \\
16 \cdot 0 \\
33 \cdot 5 \\
49 \cdot 1\end{array}$ & $\begin{array}{c}13 \cdot 7-15 \cdot 6 \\
49 \cdot 9-63 \cdot 9 \\
159 \cdot 1-171 \cdot 6 \\
18 \cdot 6-27 \cdot 8 \\
64 \cdot 3-182 \cdot 1 \\
7 \cdot 5-9 \cdot 0 \\
41 \cdot 6-70 \cdot 8 \\
6988 \cdot 5-10893 \cdot 1 \\
1671 \cdot 9-2606 \cdot 0 \\
8836 \cdot 9-11672 \cdot 2 \\
2114 \cdot 1-2792 \cdot 4 \\
13 \cdot 9-18 \cdot 3 \\
29 \cdot 7-37 \cdot 7 \\
44 \cdot 3-53 \cdot 7\end{array}$ \\
\hline Carbohydrates (\% of total El) & $n$ & $\%$ & $n$ & $\%$ & $n$ & $\%$ & $n$ & $\%$ \\
\hline \multicolumn{9}{|l|}{ Sex } \\
\hline $\begin{array}{l}\text { Boys } \\
\text { Girls }\end{array}$ & $\begin{array}{l}208 \\
296\end{array}$ & $\begin{array}{l}41 \cdot 3 \\
58 \cdot 7\end{array}$ & $\begin{array}{l}368 \\
404\end{array}$ & $\begin{array}{l}47 \cdot 7 \\
52 \cdot 3\end{array}$ & $\begin{array}{l}108 \\
128\end{array}$ & $\begin{array}{l}45 \cdot 8 \\
54 \cdot 2\end{array}$ & $\begin{array}{l}684 \\
828\end{array}$ & $\begin{array}{l}45 \cdot 2 \\
54 \cdot 8\end{array}$ \\
\hline \multicolumn{9}{|l|}{ Weight status $\dagger$} \\
\hline $\begin{array}{l}\text { Underweight } \\
\text { Normal weight } \\
\text { Overweight } \\
\text { Obese }\end{array}$ & $\begin{array}{r}14 \\
303 \\
140 \\
47\end{array}$ & $\begin{array}{r}2 \cdot 8 \\
60.1 \\
27 \cdot 8 \\
9.3\end{array}$ & $\begin{array}{r}58 \\
609 \\
83 \\
22\end{array}$ & $\begin{array}{r}7.5 \\
78.9 \\
10.8 \\
2.8\end{array}$ & $\begin{array}{r}36 \\
185 \\
13 \\
2\end{array}$ & $\begin{array}{r}15 \cdot 3 \\
78 \cdot 4 \\
5 \cdot 5 \\
0.8\end{array}$ & $\begin{array}{r}108 \\
1097 \\
236 \\
71\end{array}$ & $\begin{array}{r}7.1 \\
72 \cdot 6 \\
15 \cdot 6 \\
4.7\end{array}$ \\
\hline \multicolumn{9}{|l|}{$\begin{array}{l}\text { Adolescent's perception of mother's } \\
\text { weight }\end{array}$} \\
\hline $\begin{array}{l}\text { Normal weight/thin } \\
\text { Overweight/obese }\end{array}$ & $\begin{array}{r}391 \\
84\end{array}$ & $\begin{array}{l}82 \cdot 3 \\
17 \cdot 7\end{array}$ & $\begin{array}{r}661 \\
78\end{array}$ & $\begin{array}{l}89 \cdot 4 \\
10 \cdot 6\end{array}$ & $\begin{array}{r}217 \\
16\end{array}$ & $\begin{array}{r}93.1 \\
6.9\end{array}$ & $\begin{array}{r}1269 \\
178\end{array}$ & $\begin{array}{l}87 \cdot 7 \\
12 \cdot 3\end{array}$ \\
\hline \multicolumn{9}{|l|}{ Adolescent's perception of father's weight } \\
\hline $\begin{array}{l}\text { Normal weight/thin } \\
\text { Overweight/obese }\end{array}$ & $\begin{array}{r}373 \\
83\end{array}$ & $\begin{array}{l}81 \cdot 8 \\
18 \cdot 2\end{array}$ & $\begin{array}{l}604 \\
121\end{array}$ & $\begin{array}{l}83 \cdot 3 \\
16 \cdot 7\end{array}$ & $\begin{array}{r}194 \\
33\end{array}$ & $\begin{array}{l}85 \cdot 5 \\
14.5\end{array}$ & $\begin{array}{r}1171 \\
237\end{array}$ & $\begin{array}{l}83 \cdot 2 \\
16 \cdot 8\end{array}$ \\
\hline \multicolumn{9}{|l|}{ Mother's education } \\
\hline $\begin{array}{l}\text { education } \\
\text { High secondary education/university } \\
\text { education } \\
\text { Father's education }\end{array}$ & 309 & 64.5 & 531 & 71.7 & 156 & 68.7 & 996 & $68 \cdot 8$ \\
\hline $\begin{array}{l}\text { Lower education/lower secondary } \\
\text { education }\end{array}$ & 173 & 37.9 & 224 & $31 \cdot 1$ & 63 & $28 \cdot 4$ & 460 & $32 \cdot 9$ \\
\hline $\begin{array}{l}\text { High secondary education/university } \\
\text { education }\end{array}$ & 283 & $62 \cdot 1$ & 497 & 68.9 & 159 & $71 \cdot 6$ & 939 & $67 \cdot 1$ \\
\hline \multicolumn{9}{|l|}{ Family Affluence Scale } \\
\hline $\begin{array}{l}\text { High } \\
\text { I am very worried about gaining weight }\end{array}$ & 176 & $35 \cdot 3$ & 270 & 35.0 & $\begin{array}{r}148 \\
88\end{array}$ & $\begin{array}{l}02.1 \\
37.3\end{array}$ & $\begin{array}{l}971 \\
534\end{array}$ & $\begin{array}{l}64 \cdot 5 \\
35 \cdot 5\end{array}$ \\
\hline $\begin{array}{l}\text { I am very worried about gaining weight } \\
\text { Not at all/seldomly true } \\
\text { Sometimes/always true }\end{array}$ & $\begin{array}{l}224 \\
269\end{array}$ & $\begin{array}{l}45.4 \\
54 \cdot 6\end{array}$ & $\begin{array}{l}507 \\
258\end{array}$ & $\begin{array}{l}66 \cdot 3 \\
33 \cdot 7\end{array}$ & $\begin{array}{r}184 \\
50\end{array}$ & $\begin{array}{l}78 \cdot 6 \\
21 \cdot 4\end{array}$ & $\begin{array}{l}915 \\
577\end{array}$ & $\begin{array}{l}61 \cdot 3 \\
38 \cdot 7\end{array}$ \\
\hline $\begin{array}{l}\text { I dread being fat } \\
\text { Not at all/seldomly true } \\
\text { Sometimes/alwavs true }\end{array}$ & $\begin{array}{l}227 \\
268\end{array}$ & $\begin{array}{l}45 \cdot 9 \\
54 \cdot 1\end{array}$ & $\begin{array}{l}431 \\
332\end{array}$ & $\begin{array}{l}56 \cdot 5 \\
43.5\end{array}$ & $\begin{array}{r}147 \\
87\end{array}$ & $\begin{array}{l}62 \cdot 8 \\
37 \cdot 2\end{array}$ & $\begin{array}{l}805 \\
687\end{array}$ & $\begin{array}{l}54 \cdot 0 \\
46 \cdot 0\end{array}$ \\
\hline \multicolumn{9}{|l|}{$\begin{array}{l}\text { I am constantly aware that I weigh too } \\
\text { much }\end{array}$} \\
\hline Not at all/seldomly true & 282 & $57 \cdot 2$ & 584 & 76.6 & 191 & $82 \cdot 3$ & 1057 & 71.1 \\
\hline \multicolumn{9}{|l|}{ I often eat less than I would like } \\
\hline $\begin{array}{l}\text { Not at all/seldomly true } \\
\text { Sometimes/always true }\end{array}$ & $\begin{array}{l}337 \\
157\end{array}$ & $\begin{array}{l}68 \cdot 2 \\
31.8\end{array}$ & $\begin{array}{l}623 \\
141\end{array}$ & $\begin{array}{l}81.5 \\
18.5\end{array}$ & $\begin{array}{r}195 \\
38\end{array}$ & $\begin{array}{l}83 \cdot 7 \\
16 \cdot 3\end{array}$ & $\begin{array}{r}1155 \\
336\end{array}$ & $\begin{array}{l}77.5 \\
22.5\end{array}$ \\
\hline \multicolumn{9}{|l|}{ I deliberately have small portions } \\
\hline $\begin{array}{l}\text { Not at all/seldomly true } \\
\text { Sometimes/always true }\end{array}$ & $\begin{array}{l}351 \\
142\end{array}$ & $\begin{array}{l}71 \cdot 2 \\
28 \cdot 8\end{array}$ & $\begin{array}{l}645 \\
117\end{array}$ & $\begin{array}{l}84 \cdot 6 \\
15 \cdot 4\end{array}$ & $\begin{array}{r}206 \\
27\end{array}$ & $\begin{array}{l}88 \cdot 4 \\
11.6\end{array}$ & $\begin{array}{r}1202 \\
286\end{array}$ & $\begin{array}{l}80 \cdot 0 \\
19 \cdot 2\end{array}$ \\
\hline \multicolumn{9}{|l|}{ I try to eat as little as possible } \\
\hline $\begin{array}{l}\text { Not at all/seldomly true } \\
\text { Sometimes/always true }\end{array}$ & $\begin{array}{l}311 \\
182\end{array}$ & $\begin{array}{l}63 \cdot 1 \\
36 \cdot 9\end{array}$ & $\begin{array}{l}614 \\
149\end{array}$ & $\begin{array}{l}80 \cdot 5 \\
19.5\end{array}$ & $\begin{array}{r}201 \\
34\end{array}$ & $\begin{array}{l}85 \cdot 5 \\
14.5\end{array}$ & $\begin{array}{r}1126 \\
365\end{array}$ & $\begin{array}{l}75 \cdot 5 \\
24 \cdot 5\end{array}$ \\
\hline \multicolumn{9}{|l|}{ I am content with my figure } \\
\hline $\begin{array}{l}\text { Not at all/seldomly true } \\
\text { Sometimes/always true }\end{array}$ & $\begin{array}{l}242 \\
253\end{array}$ & $\begin{array}{l}48 \cdot 9 \\
51 \cdot 1\end{array}$ & $\begin{array}{l}205 \\
557\end{array}$ & $\begin{array}{l}26 \cdot 9 \\
73 \cdot 1\end{array}$ & $\begin{array}{r}62 \\
169\end{array}$ & $\begin{array}{l}26 \cdot 8 \\
73 \cdot 2\end{array}$ & $\begin{array}{l}509 \\
979\end{array}$ & $\begin{array}{l}34 \cdot 2 \\
65 \cdot 8\end{array}$ \\
\hline \multicolumn{9}{|l|}{ My parents think I am too fat } \\
\hline $\begin{array}{l}\text { Not at all/seldomly true } \\
\text { Sometimes/always true }\end{array}$ & $\begin{array}{r}405 \\
85\end{array}$ & $\begin{array}{l}82 \cdot 7 \\
17 \cdot 3\end{array}$ & $\begin{array}{r}709 \\
55\end{array}$ & $\begin{array}{r}92 \cdot 8 \\
7 \cdot 2\end{array}$ & $\begin{array}{r}222 \\
13\end{array}$ & $\begin{array}{r}94.5 \\
5.5\end{array}$ & $\begin{array}{r}1336 \\
153\end{array}$ & $\begin{array}{l}89 \cdot 7 \\
10 \cdot 3\end{array}$ \\
\hline \multicolumn{9}{|l|}{ I often skip breakfast } \\
\hline $\begin{array}{l}\text { Disagree } \\
\text { Agree }\end{array}$ & $\begin{array}{l}201 \\
204\end{array}$ & $\begin{array}{l}49 \cdot 6 \\
50 \cdot 4\end{array}$ & $\begin{array}{l}442 \\
203\end{array}$ & $\begin{array}{l}68.5 \\
31.5\end{array}$ & $\begin{array}{r}133 \\
59\end{array}$ & $\begin{array}{l}69 \cdot 3 \\
30 \cdot 7\end{array}$ & $\begin{array}{l}776 \\
466\end{array}$ & $\begin{array}{l}62.5 \\
37.5\end{array}$ \\
\hline
\end{tabular}

MVPA, moderate-to-vigorous physical activity; TEE, total energy expenditure; El, energy intake.

* Based on objective physical activity measurements

$\dagger \mathrm{BMl}$ categories as described by Cole et al. ${ }^{(19,20)}$. 
Table 5. Multilevel logistic regression

(Adjusted odds ratios and $95 \%$ confidence intervals for age- and sex, including random effects for study centre)

\begin{tabular}{|c|c|c|c|c|}
\hline \multirow[b]{2}{*}{ Covariates } & \multicolumn{2}{|c|}{ Under-reporters ( $n$ 504) } & \multicolumn{2}{|c|}{ Over-reporters ( $n$ 236) } \\
\hline & OR & $95 \% \mathrm{Cl}$ & OR & $95 \% \mathrm{Cl}$ \\
\hline Age (years) & 0.98 & $0.89-1.08$ & 1.02 & $0 \cdot 90-1 \cdot 16$ \\
\hline \multicolumn{5}{|l|}{ Sex } \\
\hline Male & 1.00 & & 1.00 & \\
\hline Female & 1.34 & $1.06-1.69$ & 1.06 & $0.78-1.42$ \\
\hline \multicolumn{5}{|l|}{ Weight status $\dagger$} \\
\hline Underweight & $0.46^{*}$ & $0.25-0.84$ & $2 \cdot 00^{\star}$ & $1 \cdot 27-3 \cdot 17$ \\
\hline Normal weight & 1.00 & & 1.00 & \\
\hline Overweight & $4.04^{\star}$ & $2 \cdot 91-5 \cdot 61$ & $0.44^{\star}$ & $0.23-0.82$ \\
\hline Obese & $5 \cdot 29^{*}$ & $3.02-9 \cdot 23$ & $0.23^{*}$ & $0.05-0.99$ \\
\hline \multicolumn{5}{|l|}{ Adolescent's perception of mother's weight } \\
\hline Normal weight/thin & 1.00 & & 1.00 & \\
\hline Overweight/obese & $1.69^{*}$ & $1 \cdot 20-2 \cdot 39$ & 0.81 & $0.57-1.16$ \\
\hline \multicolumn{5}{|l|}{ Adolescent's perception of father's weight } \\
\hline Normal weight/thin & 1.00 & & 1.00 & \\
\hline Overweight/obese & 1.08 & $0.78-1.47$ & 1.05 & $0.74-1.50$ \\
\hline \multicolumn{5}{|l|}{ Mother's education } \\
\hline Lower education/lower secondary education & 1.00 & & 1.00 & \\
\hline High secondary education/university education & 0.77 & $0.59-1.01$ & 0.61 & $0.34-1.07$ \\
\hline \multicolumn{5}{|l|}{ Father's education } \\
\hline Lower education/lower secondary education & 1.00 & & 1.00 & \\
\hline High secondary education/university education & 0.78 & $0.60-1.02$ & 0.82 & $0.54-1.26$ \\
\hline \multicolumn{5}{|l|}{ Family Affluence Scale } \\
\hline Low/medium & 1.00 & & 1.00 & \\
\hline High & 1.01 & $0.78-1.30$ & 1.09 & $0.79-1.50$ \\
\hline Screen time $(\min / \mathrm{d})$ & 0.998 & $0.998-1.000$ & $1.00^{\star}$ & $1.001-1.004$ \\
\hline Sleep time $(\mathrm{h} / \mathrm{d})$ & 0.90 & $0.81-1.00$ & 1.03 & $0.89-1.19$ \\
\hline MVPA (min/d) & $1.01^{*}$ & $1.01-1.02$ & 0.99 & $0.99-1.00$ \\
\hline \multicolumn{5}{|l|}{ I am very worried about gaining weight } \\
\hline Not at all/seldomly true & 1.00 & & 1.00 & \\
\hline Sometimes/always true & $2 \cdot 57^{\star}$ & 1.99 & $0.44^{\star}$ & $0.30-0.64$ \\
\hline \multicolumn{5}{|l|}{ I dread being fat } \\
\hline Not at all/seldomly true & 1.00 & & 1.00 & \\
\hline Sometimes/always true & $1.61^{*}$ & $1.26-2.07$ & $0.66^{\star}$ & $0.48-0.92$ \\
\hline \multicolumn{5}{|l|}{ I am constantly aware that I weigh too much } \\
\hline Not at all/seldomly true & 1.00 & & 1.00 & \\
\hline Sometimes/always true & $2 \cdot 79^{*}$ & $2 \cdot 13-3 \cdot 65$ & $0.57^{*}$ & $0.38-0.85$ \\
\hline \multicolumn{5}{|l|}{ I often eat less than I would like } \\
\hline Not at all/seldomly true & 1.00 & & 1.00 & \\
\hline Sometimes/always true & $2 \cdot 23^{*}$ & $1.69-2.96$ & 0.78 & $0 \cdot 52-1 \cdot 18$ \\
\hline \multicolumn{5}{|l|}{ I deliberately have small portions } \\
\hline Not at all/seldomly true & 1.00 & & 1.00 & \\
\hline Sometimes/always true & $2 \cdot 39^{\star}$ & $1 \cdot 77-3 \cdot 21$ & 0.64 & $0.40-1.03$ \\
\hline \multicolumn{5}{|l|}{ I try to eat as little as possible } \\
\hline Not at all/seldomly true & 1.00 & & 1.00 & \\
\hline Sometimes/always true & $2 \cdot 62^{*}$ & $1.97-3.48$ & $0.61^{*}$ & $0.40-0.95$ \\
\hline \multicolumn{5}{|l|}{ I am content with my figure } \\
\hline Not at all/seldomly true & 1.00 & & 1.00 & \\
\hline Sometimes/always true & $0.35^{\star}$ & $0.27-0.46$ & $1 \cdot 19$ & $0.83-1.69$ \\
\hline My parents think I am too fat & & & & \\
\hline Not at all/seldomly true & 1.00 & & 1.00 & \\
\hline Sometimes/always true & $2 \cdot 72^{*}$ & $1.88-3.94$ & 0.70 & $0 \cdot 37-1 \cdot 33$ \\
\hline I often skip breakfast & & & & \\
\hline Disagree & 1.00 & & 1.00 & \\
\hline Agree & $2 \cdot 29^{*}$ & $1.74-3.02$ & 0.84 & $0.58-1.22$ \\
\hline Proteins (\% of total El) & $1.09^{*}$ & $1.04-1 \cdot 13$ & $0.88^{\star}$ & $0.83-0.94$ \\
\hline Fat $(\%$ of total El) & $0.97^{*}$ & $0.95-0.99$ & 0.99 & $0.97-1.01$ \\
\hline Carbohydrates (\% of total El) & $1.03^{\star}$ & $1.01-1.04$ & 1.02 & $0.99-1.05$ \\
\hline
\end{tabular}

MVPA, moderate-to-vigorous physical activity; El, energy intake.

* Statistically significant OR.

† BMl categories as described by Cole et al. ${ }^{(19,20)}$.

characteristics such as age group (children $v$. adolescents) and status (self-report $v$. proxy). The degree of UR markedly increased (3\%) for the second interview in comparison with the first one. An increase of UR rates with the number of collected days in adolescents has been previously reported ${ }^{(32)}$. It is known that increasing the number of recording days provides more precise estimates of the individual dietary intake and reduces the within-person variability ${ }^{(33)}$; however, long 
Table 6. Multilevel logistic regression

$\dagger$ (Multivariable odds ratios and $95 \%$ confidence intervals including random effects for study centre)

\begin{tabular}{|c|c|c|c|c|}
\hline \multirow[b]{2}{*}{ Covariates } & \multicolumn{2}{|c|}{ Under-reporters ( $n$ 504) } & \multicolumn{2}{|c|}{ Over-reporters ( $n$ 236) } \\
\hline & OR & $95 \% \mathrm{Cl}$ & OR & $95 \% \mathrm{Cl}$ \\
\hline Age (years) & 0.95 & $0 \cdot 82-1 \cdot 10$ & 1.01 & $0 \cdot 88-1 \cdot 16$ \\
\hline \multicolumn{5}{|l|}{ Sex } \\
\hline Male & 1.00 & & 1.00 & \\
\hline Female & 1.25 & $0.85-1.84$ & $1 \cdot 18$ & $0.81-1.72$ \\
\hline \multicolumn{5}{|l|}{ Weight status $\ddagger$} \\
\hline Underweight & 0.53 & $0.26-1 \cdot 11$ & $1.67^{\star}$ & $1 \cdot 01-2 \cdot 76$ \\
\hline Normal weight & 1.00 & & 1.00 & \\
\hline Overweight & $3 \cdot 25^{\star}$ & $2 \cdot 01-5 \cdot 27$ & 0.52 & $0.26-1.03$ \\
\hline Obese & $4 \cdot 31^{*}$ & $1.92-9.65$ & $\mathrm{n} / \mathrm{a}$ & $\mathrm{n} / \mathrm{a}$ \\
\hline \multicolumn{5}{|l|}{ Adolescent's perception of mother's weight } \\
\hline Normal weight/thin & 1.00 & & 1.00 & \\
\hline Overweight/obese & 1.03 & $0.66-1.46$ & 0.77 & $0.42-1.41$ \\
\hline \multicolumn{5}{|l|}{ Mother's education } \\
\hline Lower education/lower secondary education & 1.00 & & - & \\
\hline High secondary education/university education & 0.92 & $0.60-1.41$ & & \\
\hline \multicolumn{5}{|l|}{ Father's education } \\
\hline Lower education/lower secondary education & 1.00 & & - & \\
\hline High secondary education/university education & 0.97 & $0.65-1.46$ & & \\
\hline Screen time $(\min / \mathrm{d})$ & $0.997^{\star}$ & $0.995-0.999$ & $1.003^{*}$ & $1.001-1.005$ \\
\hline Sleep time $(h / d)$ & 0.883 & $0.763-1.021$ & - & \\
\hline MVPA (min/d) & $1.02^{*}$ & $1.01-1.02$ & $0.991^{*}$ & $0.983-0.999$ \\
\hline \multicolumn{5}{|l|}{ I am very worried about gaining weight } \\
\hline Not at all/seldomly true & 1.00 & & 1.00 & \\
\hline Sometimes/always true & 1.53 & $0.99-2.37$ & $0.55^{\star}$ & $0.33-0.92$ \\
\hline \multicolumn{5}{|l|}{ I dread being fat } \\
\hline Not at all/seldomly true & 1.00 & & 1.00 & \\
\hline Sometimes/always true & 0.86 & $0.58-1.27$ & 1.01 & $0.68-1.51$ \\
\hline \multicolumn{5}{|l|}{ I am constantly aware that I weigh too much } \\
\hline Not at all/seldomly true & 1.00 & & 1.00 & \\
\hline Sometimes/always true & $1 \cdot 20$ & $0.76-1.90$ & 1.02 & $0.59-1.75$ \\
\hline \multicolumn{5}{|l|}{ I often eat less than I would like } \\
\hline Not at all/seldomly true & 1.00 & & - & \\
\hline Sometimes/always true & $1 \cdot 13$ & $0.71-1 \cdot 81$ & & \\
\hline \multicolumn{5}{|l|}{ I deliberately have small portions } \\
\hline Not at all/seldomly true & 1.00 & & 1.00 & \\
\hline Sometimes/always true & 0.98 & $0.59-1.63$ & $1 \cdot 18$ & $0 \cdot 65-2 \cdot 14$ \\
\hline \multicolumn{5}{|l|}{ I try to eat as little as possible } \\
\hline Not at all/seldomly true & 1.00 & & 1.00 & \\
\hline Sometimes/always true & $1 \cdot 21$ & $0.77-1.91$ & 0.84 & $0.48-1.48$ \\
\hline \multicolumn{5}{|l|}{ I am content with my figure } \\
\hline Not at all/seldomly true & 1.00 & & - & \\
\hline Sometimes/always true & $0.61^{*}$ & $0.41-0.89$ & & \\
\hline \multicolumn{5}{|l|}{ My parents think I am too fat } \\
\hline Not at all/seldomly true & 1.00 & & - & \\
\hline Sometimes/always true & 0.88 & $0.50-1.55$ & & \\
\hline \multicolumn{5}{|l|}{ I often skip breakfast } \\
\hline Disagree & 1.00 & & - & \\
\hline Agree & $2 \cdot 14^{*}$ & $1.53-2.99$ & & \\
\hline Proteins (\% of total El) & $1.08^{*}$ & $1.02-1.14$ & $0.87^{*}$ & $0.82-0.93$ \\
\hline Fat (\% of total El) & 0.98 & $0.95-1.00$ & - & \\
\hline Carbohydrates (\% of total EI) & $1.06^{*}$ & $1.03-1.09$ & - & \\
\hline
\end{tabular}

MVPA, moderate-to-vigorous physical activity; El, energy intake.

* Statistically significant OR.

† OR corresponds to a multivariable multilevel logistic regression analysis adjusted for sex, age and all the variables with $P<0.20$ in previous analyses entered simultaneously (Table 5).

$\ddagger \mathrm{BMI}$ categories as described by Cole et al. ${ }^{(19,20)}$.

recording periods tend to reduce the accuracy of recording by increasing fatigue and boredom ${ }^{(34)}$.

Sex

The association of sex with the occurrence of misreporting is not conclusive yet, at least in young populations ${ }^{(5,31,35,36)}$.
We failed to detect a significant association between sex and misreporting in any of its forms in our sample. Overall, studies with DLW did not find significant differences in energy reporting between male and female adults ${ }^{(1)}$. Livingstone \& Black $^{(1)}$ questioned the validity of these results, showing a higher proportion of UR among females as a result of the application of a single cut-off point for EI/BMR. Males usually 
have higher TEE than women, which involves higher EI values. Therefore, the use of a single cut-off point for all individuals would identify more girls as under-reporters. This hypothesis could explain other reported findings $(5,35,36)$.

\section{Age}

Unlike sex, the inverse association between increasing age and plausible reporting has been consistently reported ${ }^{(5,31,35,36)}$. Despite adolescents being able to report their own dietary intake, they usually show less interest, motivation and cooperation than children or younger adolescents - that is, 12-15-year-old individuals ${ }^{(37)}$. They might find the task of recalling their dietary intake irritating and tedious, thus decreasing the level of compliance, and subsequently increasing the reporting error ${ }^{(37)}$. No association with age, however, was observed in our study. Likewise, the above-mentioned theory formulated by Livingstone \& Black $^{(1)}$ could also explain the finding of higher levels of misreporting among older individuals; similar to males, younger individuals have higher TEE than older people, and consequently higher EI.

\section{Socio-economic indicators}

Our findings did not show any association between misreporting and the SES indicators examined. The existing literature is inconclusive on the association of misreporting with education levels or SES. Some studies reported no significant association $^{(5,38,39)}$, whereas others reported a positive association with either low ${ }^{(36)}$ or higher education levels ${ }^{(40)}$. Poor literacy skills might account for the higher misreporting in less-educated groups; however, several studies have indicated an association of misreporting, mainly as UR, with higher SES, potentially due to higher awareness for socially desirable responding ${ }^{(1)}$. Methodological differences in the assessment of SES across studies could account for these conflicting results ${ }^{(31)}$.

\section{Sedentary behaviours and physical activity}

The association between dietary misreporting and sedentary behaviours in adolescents has not been evaluated yet by other studies. We observed that adolescents with higher screen time were less likely to under-report and more likely to over-report. Lioret et $a l .{ }^{(5)}$ reported a positive association of UR with sedentary behaviours among children aged 3-10 years, with data reported mainly by the parents. Misreporting could be affected by difficulties of the individual to perform the task, inattention to eating and/or social desirability bias, among other factors $^{(37)}$. Inattentive respondents or those concerned about any social judgement may misreport EI, screen time or both at the same time, which could have occurred in our sample, but also, to certain extent, in the sample from Lioret et al. ${ }^{(5)}$. In addition, these factors affecting energy reporting could be more or less prevalent depending on the respondent status - that is, parents/proxy-reported data $v$. adolescents/self-reported data and therefore have an influence on the direction of the observed associations.
Consistent with previous literature ${ }^{(40)}$, our results showed that those engaging in higher MVPA levels were more likely to under-report their EI and less likely to be over-reporters. Similar findings were observed when moderate PA and vigorous PA were assessed separately (data not shown). One potential explanation is that those engaging in more exercise tend to have generally higher health consciousness, and may therefore be more prone to provide socially desirable responses. This could additionally explain a lower engagement in sedentary activities as a result of a major concern to have a healthy lifestyle. Higher PA performance together with lower levels of sedentary behaviours could also reflect attempts to lose weight. Hare et $a l .{ }^{(38)}$, in contrast, observed that minutes of MVPA were lower among under-reporters, although they did not manage to give a strong explanation for these findings.

\section{$B M /$}

The most robust finding in a comprehensive review of characteristics associated with misreporting ${ }^{(1)}$ was the positive association with BMI that was already reported in a number of studies $^{(5,35,36,38-40)}$. Forrestal $^{(31)}$ also observed that weight status was consistently associated with UR in both children and adolescents. Likewise, the probability of UR among the HELENA-CSS adolescents increased as BMI increased. In agreement with Rangan et al. ${ }^{(35)}$, over-reporters were more likely to be underweight. These results might be explained by social desirability bias, which could be most marked among overweight and/or obese adolescents, but might also indicate a poor ability or denial for self-monitoring of dietary intake within this group ${ }^{(35)}$. Another plausible hypothesis could be that overweight/obese people are on a diet, and thus they are truthful when reporting low EI. Weight status is indisputably a factor that needs to be taken into consideration when assessing diet-disease associations. Nonetheless, it should be noted that there are overweight and obese individuals who do not under-report, thus being reliable reporters, whereas there are under-reporters among those within normal weight range ${ }^{(1)}$. Although UR was most prevalent among overweight and obese adolescents, we noted that normal-weight and underweight adolescents accounted for $62.9 \%$ of the individuals within our UR group. On the other hand, the degree of OV among overweight and obese adolescents was extremely low (6.3\%).

\section{Figure perception and diet-related attitudes}

As already acknowledged by previous studies ${ }^{(5,6)}$, UR may not exclusively reflect UR but also real under-eating as an attempt to lose or not to gain weight. In addition, adolescents are at an age when growth spurts occur and might indeed consume large amounts of foods; this would reflect real over-eating rather than OV. Owing to the short period of dietary recording, we could not distinguish between under-reporters/over-reporters and undereaters/over-eaters. Our initial multilevel regression analysis showed that those who were more frequently concerned about gaining weight and being fat and more prone to dietary restraints by eating less to avoid gaining weight were more likely to UR and less likely to OV. In addition, those with figure dissatisfaction 
were more likely to under-report. Previous findings in adolescents have already illustrated that under-reporters exhibited greater weight consciousness and dieting ${ }^{(5,41,42)}$. Nevertheless, significant associations with weight concern and dietary restraint did not remain significant when all the variables were entered into the same model, apart from the inverse association between being concerned about gaining weight and OV and between figure satisfaction and UR. That might be partly explained by the fact that other covariates - which could be confounding the initially observed associations - that is, weight status and/or body image satisfaction - were entered into the model. This suggests that overweight/obese adolescents and/or those with poor body image could be more concerned about their weight and/or might engage in dietary restrictive behaviours more often, resulting in intentional alteration of the diet by eating less or avoiding certain food items (under-eating), and therefore less likely to OV, together with higher PA levels and lower engagement in sedentary behaviours.

Livingstone \& Robson ${ }^{(9)}$ hypothesised that obese adolescents may feel even more stigmatised about their fatness than obese adults, given the widespread and excessive obsession with body weight and image among adolescents. Consistent with the findings of Lioret et al. ${ }^{(5)}$, breakfast skipping was also more prevalent among under-reporters, which might reflect intentional dieting and further emphasise the need of differentiating among those who are truly under-reporting and those who are under-eating ${ }^{(5)}$.

Our study contributes to the existing evidence that errors in EI reporting may reflect pressure to meet cultural expectations ${ }^{(1,43)}$. It is of concern that this phenomenon might be more marked among adolescents as they are sensitive to context and social norms ${ }^{(44)}$. Attitudes towards food consumption are affected by a number of factors such as weight status and consciousness, body image, social desirability and dietary restraint ${ }^{(45)}$, which cannot be ignored when evaluating energy reporting. Thus, inaccurate reporting is not simply a nutritionists' issue; a multidisciplinary approach (including psychology, sociology and physiology) is required to further understand misreporting in dietary intake studies ${ }^{(6)}$.

\section{Macronutrient intakes}

Misreporting is not limited only to EI but may also affect the macronutrient composition of the diet. Our results showed that under-reporters had a higher contribution of protein to EI, whereas it was lower among over-reporters, as described in other studies conducted in both adolescents ${ }^{(5,46)}$ and adults $^{(1,3,6)}$. A lower percentage of fat contribution to EI has also been reported, whereas the contribution of carbohydrates to EI has been observed to be variable ${ }^{(1)}$. No significant associations, however, were observed in our study between percentage of EI from fat and misreporting, whereas adolescents with higher contribution of carbohydrates to EI were more likely to underreport. A potential explanation is that under-reporters may omit certain foods that are considered unhealthy such as sugar-rich products and/or that they restrain themselves from eating them $^{(6)}$. All these data suggest that misreporting may introduce bias in the assessment of macronutrient composition of the diet in adolescents and that should be considered when addressing relationships with macronutrient intake.

Bias in estimating nutrient intakes and in reporting meal patterns and foods eaten are the consequences of dietary misreporting, mainly due to UR, which is more widespread than $\mathrm{OV}^{(31)}$. This bias has a number of implications for the interpretation of descriptive analyses and diet-disease links. Misreporting, more specifically UR, can overestimate the number of subjects with deficient nutrient intakes, attenuate or even reverse associations between diet and disease and seriously hinder the derivation of food-based dietary guidelines due to selective reporting of foods $^{(1)}$. A common practice to deal with these concerns is to exclude UR from the analysis; however, this might exclude individuals who report low EI as a result of intentional dietary restraint or dieting - that is, under-eating ${ }^{(4)}$. As exclusion of under-reporters introduces unknown bias, under-reporters should therefore be identified and corrected for ${ }^{(47)}$. Nevertheless, it would be advisable to perform the analysis including all the subjects available in the sample with and without adjustment for either reporting status or variables associated with misreporting. Running the analysis with and without under-reporters and comparing the obtained results with each approach could be another alternative. Unfortunately, there is no formal procedure to handle misreporting in the analysis and disadvantages are present in all the approaches. However, misreporting needs to be accounted for, at least in the interpretation and discussion of results. A similar approach could also be followed with over-reporters, although among adolescents OV should be treated cautiously as higher EI may represent real overeating to cover the high energy costs due to growth.

In addition, the factors identified as major misreporting correlates in this study - that is, weight status, dietary restraint attitudes and weight dissatisfaction - could assist researchers in identifying individuals more prone to provide biased dietary intake reports; taking them into account during study design and data collection could minimise the error associated with self-reported dietary data. Future research lines including dietary intake estimates and/or addressing associations in which obesity indicators and/or dietary attitudes play an essential role will mainly benefit from these findings.

\section{Limitations and strengths}

The limitations of our study should be acknowledged. Diet was assessed by self-reported 24-HDR, which is prone to portion size estimation errors and recording bias. Dietary intake was estimated based on two non-consecutive 24-HDR, which are not sufficient to characterise individuals' usual intakes. Collection of dietary data for $>2 \mathrm{~d}$ would have been desirable to capture usual intakes and to account for day-to-day variability $^{(33)}$. However, the HELENA-DIAT has already been shown to provide reliable estimates of dietary intake among European adolescents ${ }^{(25,27)}$.

Another limitation is that the applied cut-off points assumed stable body weight, which might not always be true in growing adolescents. However, as energy costs for growth in adolescence are small, at approximately $1 \%$ of $\mathrm{TEE}^{(48)}$, we presume that this assumption does not significantly affect the study outcomes. 
Reports of EI are always subject to the possibility of undereating and over-eating. Similar to the Goldberg equation, the approach applied does not account for differences between respondents who are on a diet (under-eating) or those limiting their reported intake (UR) and among those who are intentionally eating more (over-eating) or reporting higher EI (OV). Nevertheless, aspects on the association between misreporting, mainly UR, and diet-related attitudes were also assessed to investigate under-eating in our sample.

Another factor to consider is the role of body composition, which was not investigated in our large sample of youths. In adults, free-living EI was found to be related to fat-free mass rather than to BMI or fat mass ${ }^{(49)}$. Therefore, at any given BMI, a fatter person, that is, one with lower percentage of fat-free mass, might actually have a lower EI and not be UR. Nevertheless, studies evaluating the validity of dietary assessments using the DLW found that the likelihood of UR in adolescents was most strongly predicted by higher percent body fat ${ }^{(50,51)}$.

The cross-sectional nature of this study provides a transversal perspective of correlates of dietary misreporting and cannot be used to establish causation. Finally, these findings should be interpreted from an explorative point of view, given that the level of statistical significance was set at $P \leq 0.05$ despite performing multiple comparisons.

Our study has several strengths. First, the sample guaranteed a large geographical spread all over Europe and all measurements followed standardised procedures throughout the different study centres. Cut-off values to identify misreporting were calculated for each individual based on his or her own PA levels, which resulted in a more accurate classification in reported EI. Although there is no simple approach to deal with reporting error, the method suggested by Huang et al. ${ }^{(28)}$ offered a simpler and more individualised alternative than other existing methods. In addition, the use of the calculated cut-off points made it possible to overcome some of the limitations associated with the use of the Goldberg cut-off point, such as the error linked to the estimation of $\mathrm{BMR}^{(52)}$.

\section{Conclusions}

Our study showed that EI misreporting in adolescents seems to be associated with several characteristics, specifically weight status, being worried about gaining weight, body image dissatisfaction and skipping breakfast. Our results also confirm the general finding that overweight/obese people are more likely to report implausibly low EI than normal-weight people. To the degree that this reflects UR of actual EI, it might be due to a tendency to provide socially desirable answers. This interpretation is supported by our findings that adolescents with dietary restraint and self-image dissatisfaction were more likely to underreport, which emphasises the need to identify these individuals in epidemiological studies. The potentially limited ability of the adolescents to accurately report their own dietary intakes could also explain part of our findings. Another possibility is that overweight/obese people may be dieting to control weight and may be accurately reporting low EI. Therefore, it is difficult to distinguish under-eating from UR. Similarly, higher EI could reflect over-eating due to growth spurts instead of OV. Therefore, factors influencing misreporting, as the ones identified in our study, should be assessed in young populations to improve the interpretation of potentially biased findings, more in particular when addressing diet-obesity associations.

\section{Acknowledgements}

The authors gratefully acknowledge all participating children and adolescents and their parents and teachers for their collaboration.

The writing group takes sole responsibility for the content of this article. The content of this article reflects the views of the authors only, and the European Community is not liable for any use that may be made of the information contained therein. The HELENA-CSS study was carried out with the financial support of the European Community Sixth RTD Framework Programme (contract FOODCT-2005-007034). This work was also partially supported by the Swedish Council for Working Life and Social Research (FAS), and by grants from the Spanish Ministry of Education (EX-2007-1124), the Spanish Ministry of Health: Maternal, Child Health and Development Network (number RD08/0072). Funders had no role in the design, analysis or writing of this article.

M. G.-G., M. K., F. G., D. M., J. D., K. W., L. A. M., Y. M., S. D. H., C. L. and I. H. conceived and designed the study; S. B.-S., E. G., L. H., M. P. and R. R. conducted the research and collected data; S. B.-S. analysed the data; C. J.-A. and I. H. contributed to the analysis; S. B.-S. and I. H. wrote the paper; S. B.-S. and I. H. participated in data interpretation; S. B.-S., C. J.-A., M. G.-G., T. M., C. B., E. G., M. K., M. C.-G., F. G., D. M., L. H., J. D., M. P., R. R., K. W., L. A. M., Y. M., S. D. H., C. L., S. V., S. L., B. G. and I. H. critically reviewed the manuscript. B. G. edited the manuscript for English language usage. All the authors read and approved the final version of the manuscript.

The authors declare that there are no conflicts of interest.

\section{References}

1. Livingstone MB \& Black AE (2003) Markers of the validity of reported energy intake. J Nutr 133, Suppl. 3, 895s-920s.

2. Willett W (1987) Nutritional epidemiology: issues and challenges. Int J Epidemiol 16, 312-317.

3. Lafay L, Basdevant A, Charles MA, et al. (1997) Determinants and nature of dietary underreporting in a free-living population: the Fleurbaix Laventie Ville Sante (FLVS) Study. Int J Obes Relat Metab Disord 21, 567-573.

4. Mendez MA (2015) Invited commentary: dietary misreporting as a potential source of bias in diet-disease associations: future directions in nutritional epidemiology research. $A m J$ Epidemiol 181, 234-236.

5. Lioret S, Touvier M, Balin M, et al. (2011) Characteristics of energy under-reporting in children and adolescents. Br J Nutr 105, 1671-1680.

6. Macdiarmid J \& Blundell J (1998) Assessing dietary intake: who, what and why of under-reporting. Nutr Res Rev 11, 231-253.

7. Nielsen SJ \& Adair L (2007) An alternative to dietary data exclusions. J Am Diet Assoc 107, 792-799.

8. Börnhorst C, Huybrechts I, Ahrens W, et al. (2013) Prevalence and determinants of misreporting among European children in proxy-reported $24 \mathrm{~h}$ dietary recalls. Br J Nutr 109, $1257-1265$. 
9. Livingstone MB \& Robson PJ (2000) Measurement of dietary intake in children. Proc Nutr Soc 59, 279-293.

10. Moreno LA, Gonzalez-Gross M, Kersting M, et al. (2008) Assessing, understanding and modifying nutritional status, eating habits and physical activity in European adolescents: the HELENA (Healthy Lifestyle in Europe by Nutrition in Adolescence) Study. Public Health Nutr 11, 288-299.

11. Moreno LA, De Henauw S, Gonzalez-Gross M, et al. (2008) Design and implementation of the Healthy Lifestyle in Europe by Nutrition in Adolescence Cross-Sectional Study. Int J Obes (Lond) 32, Suppl. 5, S4-S11.

12. Beghin L, Castera M, Manios Y, et al. (2008) Quality assurance of ethical issues and regulatory aspects relating to good clinical practices in the HELENA Cross-Sectional Study. Int J Obes 32, Suppl. 5, S12-S18.

13. Iliescu C, Beghin L, Maes L, et al. (2008) Socioeconomic questionnaire and clinical assessment in the HELENA CrossSectional Study: methodology. Int J Obes (Lond) 32, Suppl. 5, S19-S25.

14. Currie C, Molcho M, Boyce W, et al. (2008) Researching health inequalities in adolescents: the development of the Health Behaviour in School-Aged Children (HBSC) family affluence scale. Soc Sci Med 66, 1429-1436.

15. Rey-Lopez JP, Vicente-Rodriguez G, Ortega FB, et al. (2010) Sedentary patterns and media availability in European adolescents: The HELENA study. Prev Med 51, 50-55.

16. Ruiz JR, Ortega FB, Martinez-Gomez D, et al. (2011) Objectively measured physical activity and sedentary time in European adolescents: the HELENA study. Am J Epidemiol 174, 173-184.

17. Ekelund U, Sjostrom M, Yngve A, et al. (2001) Physical activity assessed by activity monitor and doubly labeled water in children. Med Sci Sports Exerc 33, 275-281.

18. Nagy E, Vicente-Rodriguez G, Manios Y, et al. (2008) Harmonization process and reliability assessment of anthropometric measurements in a multicenter study in adolescents. Int J Obes (Lond) 32, Suppl. 5, S58-S65.

19. Cole TJ, Bellizzi MC, Flegal KM, et al. (2000) Establishing a standard definition for child overweight and obesity worldwide: international survey. BMJ 320, 1240-1243.

20. Cole TJ, Flegal KM, Nicholls D, et al. (2007) Body mass index cut offs to define thinness in children and adolescents: international survey. BMJ 335, 194.

21. Diehl JM (1999) [Attitude to eating and body weight by 11- to 16-year-old adolescents]. Schweiz Med Wochenschr 129, $162-175$.

22. Kersting M, Sichert-Hellert W, Vereecken CA, et al. (2008) Food and nutrient intake, nutritional knowledge and dietrelated attitudes in European adolescents. Int J Obes (Lond) 32, Suppl. 5, S35-S41.

23. Gilbert CC, Hall G, Hegyi A, et al. (2013) Food choices and preferences. In HELENA Cross-Sectional Study Manual of Operations, pp. 101-109 [M Gonzalez-Gross, S De Henauw, F Gottrand, CC Gilbert and L Moreno, editors]. Zaragoza: University of Zaragoza.

24. Biro G, Hulshof KF, Ovesen L, et al. (2002) Selection of methodology to assess food intake. Eur J Clin Nutr 56, Suppl. 2, S25-S32.

25. Vereecken CA, Covents M, Sichert-Hellert W, et al. (2008) Development and evaluation of a self-administered computerized 24-h dietary recall method for adolescents in Europe. Int J Obes (Lond) 32, Suppl. 5, S26-S34.

26. Dehne LI, Klemm C, Henseler G, et al. (1999) The German Food Code and Nutrient Data Base (BLS II.2). Eur J Epidemiol 15, 355-359.
27. Julian-Almarcegui C, Bel-Serrat S, Kersting M, et al. (2015) Comparison of different approaches to calculate nutrient intakes based upon 24-h recall data derived from a multicenter study in European adolescents. Eur J Nutr (epublication ahead of print version 10 March 2015).

28. Huang TT, Roberts SB, Howarth NC, et al. (2005) Effect of screening out implausible energy intake reports on relationships between diet and BMI. Obes Res 13, 1205-1217.

29. Garriguet D (2008) Impact of identifying plausible respondents on the under-reporting of energy intake in the Canadian Community Health Survey. Health Rep 19, 47-55.

30. Black AE \& Cole TJ (2000) Within- and between-subject variation in energy expenditure measured by the doubly-labelled water technique: implications for validating reported dietary energy intake. Eur J Clin Nutr 54, 386-394.

31. Forrestal SG (2011) Energy intake misreporting among children and adolescents: a literature review. Matern Child Nutr 7, 112-127.

32. Moreno LA, Kersting M, de Henauw S, et al. (2005) How to measure dietary intake and food habits in adolescence: the European perspective. Int J Obes (Lond) 29, Suppl. 2, S66-S77.

33. Thompson F \& Subar A (2008) Dietary assessment methodology. In Nutrition in the Prevention and Treatment of Disease, 2nd ed. pp. 3-39 [A Coulston and C Boushey, editors]. San Diego, CA: Elsevier Academic Press.

34. Gibson RS (1987) Sources of error and variability in dietary assessment methods: a review. J Can Diet Assoc 48, 150-155.

35. Rangan AM, Flood VM \& Gill TP (2011) Misreporting of energy intake in the 2007 Australian Children's Survey: identification, characteristics and impact of misreporters. Nutrients $\mathbf{3}$, 186-199.

36. Murakami K, Miyake Y, Sasaki S, et al. (2012) Characteristics of under- and over-reporters of energy intake among Japanese children and adolescents: The Ryukyus Child Health Study. Nutrition 28, 532-538.

37. Collins CE, Watson J \& Burrows T (2010) Measuring dietary intake in children and adolescents in the context of overweight and obesity. Int J Obes (Lond) 34, 1103-1115.

38. Hare ME, Sherrill-Mittleman D, Klesges RC, et al. (2012) Energy underreporting in African-American girls: a longitudinal analysis. Child Obes (Lond) 8, 551-560.

39. Lanctot JQ, Klesges RC, Stockton MB, et al. (2008) Prevalence and characteristics of energy underreporting in AfricanAmerican girls. Obesity (Silver Spring) 16, 1407-1412.

40. Garriguet D (2008) Under-reporting of energy intake in the Canadian Community Health Survey. Health Rep 19, 37-45.

41. Livingstone MB, Robson PJ \& Wallace JM (2004) Issues in dietary intake assessment of children and adolescents. $\mathrm{Br} \mathrm{J}$ Nutr 92, Suppl. 2, S213-S222.

42. Ventura AK, Loken E, Mitchell DC, et al. (2006) Understanding reporting bias in the dietary recall data of 11-year-old girls. Obesity (Silver Spring) 14, 1073-1084.

43. Rennie KL, Siervo M \& Jebb SA (2006) Can self-reported dieting and dietary restraint identify underreporters of energy intake in dietary surveys? J Am Diet Assoc 106, 1667-1672.

44. Borgers N, de Leeuw E \& Hox J (2000) Children as respondents in survey research: cognitive development and response quality. Bull Methodol S 66, 60-75.

45. Baranowski T, Cullen KW \& Baranowski J (1999) Psychosocial correlates of dietary intake: advancing dietary intervention. Annu Rev Nutr 19, 17-40.

46. Kobe H, Krzisnik C \& Mis NF (2012) Under- and overreporting of energy intake in Slovenian adolescents. $J$ Nutr Educ Behav 44, 574-583.

47. European Food Safety Authority (2014) Guidance on the EU menu methodology. EFSA J 12, 3944. 
48. Food and Agricultural Organisation, World Health Organisation \& United Nations University (2004) Report on Human Energy Requirements; Report of the Joint FAO/WHO/UNU Expert Consultation Technical Report Series no. XX. Rome: FAO.

49. Blundell JE, Caudwell P, Gibbons C, et al. (2012) Body composition and appetite: fat-free mass (but not fat mass or BMI) is positively associated with self-determined meal size and daily energy intake in humans. Br J Nutr 107, 445-449.

50. Bratteby LE, Sandhagen B, Fan H, et al. (1998) Total energy expenditure and physical activity as assessed by the doubly labeled water method in Swedish adolescents in whom energy intake was underestimated by 7-d diet records. $A m J$ Clin Nutr 67, 905-911.

51. Perks SM, Roemmich JN, Sandow-Pajewski M, et al. (2000) Alterations in growth and body composition during puberty. IV. Energy intake estimated by the youth-adolescent food-frequency questionnaire: validation by the doubly labeled water method. Am J Clin Nutr 72, 1455-1460.

52. McCrory MA, McCrory MA, Hajduk CL, et al. (2002) Procedures for screening out inaccurate reports of dietary energy intake. Public Health Nutr 5, 873-882.

\section{Appendix: HELENA study group}

\section{Coordinator}

L. A. M.

\section{Core group members}

L. A. M., F. G., S. D. H., M. G.-G. and C. Gilbert.

\section{Steering committee}

A. Kafatos (President), L. A. M., C. Libersa, S. D. H., J. Sánchez, F. G., M. K., M. Sjöstrom, D. M., M. G.-G., J. D., C. Gilbert, G. Hall, L. Maes and L. Scalfi.

\section{Project manager}

P. Meléndez.

\section{Universidad de Zaragoza (Spain)}

L. A. M., J. Fleta, J. A. Casajús, G. Rodríguez, C. Tomás, M. I. Mesana, G. Vicente-Rodríguez, A. Villarroya, C. M. Gil, I. Ara, J. Revenga, C. Lachen, J. Fernández-Alvira, G. Bueno, A. Lázaro, O. Bueno, J. F. León, J. M. Garagorri, M. Bueno, J. P. Rey López, I. Iglesia, P. Velasco, S. B.-S., L. Gracia-Marco, T. M. and D. Jiménez-Pavón.

\section{Consejo Superior de Investigaciones Científicas (Spain)}

A. Marcos, J. Wärnberg, E. Nova, S. Gómez-Martínez, E. Ligia Díaz, J. Romeo, A. Veses, M. Angeles Puertollano, B. Zapatera and T. Pozo.

\section{Université de Lille 2 (France)}

L. Beghin, C. Libersa, F. G., C. Iliescu and J. Von Berlepsch.
Research Institute of Child Nutrition Dortmund, Rheinische Friedrich-Wilhelms-Universität Bonn (Germany)

M. K., W. Sichert-Hellert and E. Koeppen.

Pécsi Tudományegyetem (University of Pécs) (Hungary)

D. M., E. Erhardt, K. Csernus, K. Török, S. Bokor, Angster, E. Nagy, O. Kovács and J. Répasi.

\section{University of Crete School of Medicine (Greece)}

A. Kafatos, C. Codrington, M. P., A. Papadaki, K. Sarri, A. Viskadourou, C. Hatzis, M. Kiriakakis, G. Tsibinos, C. Vardavas, M. Sbokos, E. Protoyeraki and M. Fasoulaki.

Institut für Ernährungs- und Lebensmitte/wissenschaften Ernährungsphysiologie. Rheinische Friedrich-WilhelmsUniversität (Germany)

P. Stehle, K. Pietrzik, M. G.-G., C. Breidenassel, A. Spinneker, J. Al-Tahan, M. Segoviano, A. Berchtold, C. Bierschbach, E. Blatzheim, A. Schuch and P. Pickert.

\section{University of Granada (Spain)}

M. J. Castillo, A. Gutiérrez, F. B. Ortega, J. R. Ruiz, E. G. Artero, V. España, D. Jiménez-Pavón, P. Chillón, C. Sánchez-Muñoz and M. C.-G.

Council for Agricultural Research and Economics (CREA), Research Center for Food and Nutrition, Rome, Italy

D. Arcella, E. Azzini, E. Barrison, N. Bevilacqua, P. Buonocore, G. Catasta, L. Censi, D. Ciarapica, P. D'Acapito, M. Ferrari, M. Galfo, C. Le Donne, C. L., G. Maiani, B. Mauro, L. Mistura, A. Pasquali, R. Piccinelli, A. Polito, R. R., R. Spada, S. Sette and M. Zaccaria.

University of Napoli 'Federico II' Department of Food Science (Italy)

L. Scalfi, P. Vitaglione and C. Montagnese.

\section{Ghent University (Belgium)}

I. De Bourdeaudhuij, S. D. H., T. De Vriendt, L. Maes, C. Matthys, C. Vereecken, M. de Maeyer, C. Ottevaere and I. H.

\section{Medical University of Vienna (Austria)}

K. W., K. Phillipp, S. Dietrich, B. Kubelka and M. Boriss-Riedl.

\section{Harokopio University (Greece)}

Y. M., E. G., Z. Bouloubasi, T. Louisa Cook, S. Eleutheriou, O. Consta, G. Moschonis, I. Katsaroli, G. Kraniou, S. Papoutsou, 
D. Keke, I. Petraki, E. Bellou, S. Tanagra, K. Kallianoti, D. Argyropoulou, K. Kondaki, S. Tsikrika and C. Karaiskos.

Institut Pasteur de Lille (France)

J. D. and A. Meirhaeghe.

Karolinska Institutet (Sweden)

M. Sjöström, J. Ruiz, F. B. Ortega, M. Hagströmer, L. H., E. Patterson, L. Kwak, J. Wärnberg, N. Rizzo and A. Hurtig Wennlöf.

Asociación de Investigación de la Industria Agroalimentaria (Spain)

J. Sánchez-Molero, E. Picó, M. Navarro, B. Viadel, J. E. Carreres, G. Merino, R. Sanjuán, M. Lorente, M. J. Sánchez and S. Castelló.

\section{Campden BRI (UK)}

C. Gilbert, S. Thomas, E. Allchurch and P. Burguess.

SIK-Institutet foer Livsmedel och Bioteknik (Sweden)

G. Hall, A. Astrom, A. Sverkén and A. Broberg.

Meurice Recherche \& Development asbl (Belgium)

A. Masson, C. Lehoux, P. Brabant, P. Pate and L. Fontaine.
Campden \& Chorleywood Food Development Institute (Hungary)

A. Sebok, T. Kuti and A. Hegyi.

Productos Aditivos SA (Spain)

C. Maldonado and A. Llorente.

Cárnicas Serrano SL (Spain)

E. García.

Cederroth International $A B$ (Sweden)

H. von Fircks, M. Lilja Hallberg and M. Messerer.

Lantmännen Food R\&D (Sweden)

M. Larsson, H. Fredriksson, V. Adamsson and I. Börjesson.

European Food Information Council (Belgium)

L. Fernández, L. Smillie and J. Wills.

Universidad Politécnica de Madrid (Spain)

M. G.-G., J. Valtueña, U. Albers, R. Pedrero, A. Meléndez, P. J. Benito, D. Cañada, D. Jiménez-Pavón, A. Urzanqui, J. C. Ortiz, F. Fuentes, J. J. Gómez Lorente, R. Mardía Torres and P. Navarro. 